\title{
BMJ Open Does the use of patient decision aids lead to cost savings? a systematic review
}

\author{
Peter Scalia (D) , ${ }^{1}$ Paul J Barr, ${ }^{1}$ Ciaran O'Neill, ${ }^{2}$ Grainne E Crealey, ${ }^{3}$ \\ Pamela J Bagley, ${ }^{4}$ Heather B Blunt, ${ }^{4}$ Glyn Elwyn (D) ${ }^{1}$
}

To cite: Scalia P, Barr PJ, O'Neill C, et al. Does the use of patient decision aids lead to cost savings? a systematic review. BMJ Open 2020;10:e036834. doi:10.1136/ bmjopen-2020-036834

\section{- Prepublication history and} additional material for this paper are available online. To view these files, please visit the journal online (http://dx.doi. org/10.1136/bmjopen-2020036834).

Received 06 January 2020 Revised 15 August 2020 Accepted 17 September 2020

Check for updates

(c) Author(s) (or their employer(s)) 2020. Re-use permitted under CC BY-NC. No commercial re-use. See rights and permissions. Published by BMJ.

${ }^{1}$ The Dartmouth Institute for Health Policy and Clinical Practice, Dartmouth College, Lebanon, New Hampshire, USA

${ }^{2}$ Centre for Public Health,

Queen's University Belfast, Belfast, UK

${ }^{3}$ Clinical Costing Solutions, Belfast, UK

${ }^{4}$ Dartmouth College Biomedical Libraries, Hanover, New Hampshire, USA

Correspondence to

Dr Glyn Elwyn;

glynelwyn@gmail.com

\section{ABSTRACT}

Objectives To update a previous systematic review to determine if patient decision aid (PDA) interventions generate savings in healthcare settings, and if so, from which perspective (ie, patient, organisation providing care, society).

Design Systematic review.

Data sources MEDLINE, CINAHL, PsycINFO, Web of Science, Cochrane Library, Embase, Campbell Collaboration Library, EconLit, Business Source Complete, Centre for Reviews and Dissemination: NHS Economic Evaluations Database (NHS EED), Database of Abstracts of Reviews of Effects (DARE) and Health Technology Assessment (HTA) from 15 March 2013 to 25 January 2019. The references of studies that met the eligibility criteria and any publications related to conference abstracts or registered clinical trials were reviewed to increase the sensitivity of the search.

Eligibility criteria Full and partial economic evaluations with an experimental, quasi-experimental or randomised controlled design were included. The intervention had to satisfy the pre-determined minimum conditions necessary to be defined as a PDA, and (for full evaluations) provide details on the comparator used.

Data extraction and synthesis All study outcomes and economic data were extracted. The reporting and quality of the economic analyses were independently assessed by two health economists.

Results Of 5066 studies, 22 studies were included, including the 8 studies from the previous review. Twelve studies reported cost-savings (range=US $\$ 10$ to US $\$ 81$ 156; US dollars in 2020), primarily from the organisational or health system perspective, and 10 studies did not. However, due to the quality of the economic analyses, and the related issues with the interpretative validity of results it would be inappropriate to say that PDAs will generate savings, from any perspective.

Conclusions It is unclear whether PDAs will generate savings. Greater consensus on what constitutes a PDA and the need to compare them against usual care over a sufficient time horizon to allow valid assessment of costs and outcomes is required.

PROSPERO registration number CRD42019118457.

\section{INTRODUCTION}

The use of patient decision aids (PDAs) tends to shift patients' preference towards either non-surgical interventions or more towards risk-averse treatment options and this has
Strengths and limitations of this study

A strength of this study was the adherence to PRISMA (Preferred Reporting Items for Systematic Reviews and Meta-Analyses) reporting and best practice guidelines for conducting a systematic review.

- We employed a very broad inclusion criteria to provide more insight into the challenges of undertaking economic evaluations of patient decision aids.

- Two health economists independently assessed the reporting and quality of the economic analyses using two checklists and evaluated the interpretative validity of results.

- A weakness of our systematic review is that the intervention descriptions often lacked sufficient detail, so it was difficult to determine if they met the definition of a patient decision aid.

- The heterogeneity of the methods and results did not enable a meta-analysis.

led to the presumption that these tools lead to cost-savings. ${ }^{1}$ We define 'cost-saving' as a positive net monetary benefit to an alternative. A previous systematic review found that the evidence to support that claim was weak: half of the eight economic analyses included found that PDAs generated significant savings, but they were considered to be of low or moderate quality. ${ }^{2}$ Since then, PDA research has increasingly focussed on the implementation of these tools in clinic workflow, offering more opportunities to study their effects on cost from various perspectives. ${ }^{3-5}$ What conclusions can we draw from reviewing the totality of the evidence regarding the effects of PDAs on cost in healthcare settings?

PDAs provide evidence-based information in a comparative format to help patients make decisions that align with their preferences. ${ }^{1}$ Randomised trials have shown that these tools have increase knowledge and awareness of treatment options, engagement in the decision-making process, improve risk perception and reduce decisional conflict. ${ }^{1}$ A couple of systematic reviews have focussed on the cost-effectiveness of PDAs in clinical 
practice. ${ }^{6}$ In 2014, Trenaman et $a l^{7}$ found considerable variation regarding the costs of administering PDAs. The review concludes that encouraging PDA implementation to reduce spending is 'inappropriate' considering that short-term costs incurred may actually be higher when using a tool, with only one randomised trial providing evidence for cost-savings beyond 1 year. ${ }^{7}$ This evidence echoes Légaré $e t a l^{6}$ which found that there was insufficient information (or uncertainty) to make any claims regarding costs.

Assessing whether or not PDAs generate savings is challenging not just due to the lack of methodological rigour (eg, insufficient length of follow-up which is typically 2 years) or high risk of bias in studies, but because there is no consensus on an economic evaluative framework. ${ }^{8}$ Butt $^{8}$ argues that the absence of an established framework and disagreement on how researchers attribute value (monetary or otherwise) to PDAs has left us unable to draw any meaningful conclusions.

Since our previous systematic review, more economic analyses of PDAs have been conducted which may potentially provide insight into whether or not these tools generate savings-a question that is increasingly relevant for policymakers and organisations who wish to implement these tools to improve healthcare communication and delivery. Our aim in this study was to update the previous systematic review ${ }^{2}$ to determine if PDA interventions generate savings in healthcare settings, and if so, from which perspective (ie, patient, organisation providing care, society).

\section{METHODS}

We updated a previous systematic review ${ }^{2}$ according to Preferred Reporting Items for Systematic Reviews and Meta-Analyses (PRISMA) guidelines (online supplemental material table A) - PROSPERO - registration \# CRD42019118457, 21 January 2019.

\section{Inclusion criteria}

We employed the population, intervention, control, outcomes, study design (PICOS) criteria to assess study eligibility with no restrictions. ${ }^{9}$ To be eligible for inclusion, studies had to:

i. meet the following definition of decision support intervention-a definition that is synonymous with PDA: tools that "help people make specific and deliberative choices among options (including the status quo) by providing (at minimum) information on the options and outcomes relevant to a person's health status and implicit methods to clarify values". We expanded the intervention criteria to include tools that provide personalised patient probabilities of an event or outcome (eg, the risk of stroke or death for an individual based on their family history, age and so on). Expanding the inclusion criteria enables us to provide additional insight into the challenges of undertaking economic evaluations of patient decisions, as reported by Ara $e t a l^{10}$ as part of the Policy Research Unit in Economic Evaluation of Health and Care Interventions (EEPRU) 2015 report. The EEPRU report undertook research to develop a framework to evaluate the economics associated with the use of PDAs. ${ }^{10}$ They limited their review only to those studies which assessed both the costs and benefits associated with any shared decision-making process involving PDAs in any indication or setting (ie, limiting the search to only full economic evaluations). ${ }^{10}$ The emergence of both physiological and preferencebased personalised healthcare has questioned whether this conventional economic evaluation framework is sufficient to capture a range of non-health benefits and process outcomes which are emerging as key drivers of 'value'. Given that the true 'value' of PDAs most likely extends beyond this conventional paradigm, we employed a broad definition to capture not only full evaluations, but also partial evaluations, and other studies which have measured the resource implications of involving patients in shared decision making; ${ }^{10}$

ii. include a control group such as usual care, the absence of a PDA that meets the above definition, or an alternative PDA;

iii. meet our expanded study design criteria to include randomised controlled trials (RCTs), economic evaluations, observational and experimental or quasiexperimental designs which contained a control group, partial economic evaluations where patients may have acted as their own control (pre-post studies) or where the authors appealed to evidence of 'no difference in effect' and looked at differences in cost between a control and intervention group, and economic studies that used trial data (eg, an economic evaluation conducted alongside an RCT);

iv. specify the primary secondary outcomes.

We excluded Markov models and economic models that used computer-simulated data, hypothetical data or data estimates based on expert opinion because they often lack transparency, or the quality of such data may be open to debate. Including these models may have also led to 'double-counting' if the data used to populate the model is derived from a trial that is already included in our review.

\section{Data sources and search strategy}

Two information scientists (PJB and HBB) updated the previous systematic review ${ }^{2}$ search strategy and adapted it for the following databases: MEDLINE, CINAHL, PsycINFO, Web of Science, Cochrane Library, Embase, Campbell Collaboration Library, EconLit, Business Source Complete, Centre for Reviews and Dissemination: NHS Economic Evaluations Database (NHS EED), Database of Abstracts of Reviews of Effects (DARE) and Health Technology Assessment (HTA). The previous review searched databases from their inception to 15 March 2013. Therefore, in this update, the search dates 
covered 15 March 2013 to 25 January 2019 and identified studies related to: decision support interventions/PDAs and cost benefit (online supplemental material table B). We did not impose language restrictions. We reviewed the references of included studies and also searched for related conference abstracts or registered clinical trials.

\section{Study selection}

After removing duplicate study titles, PS reviewed the titles and abstracts of identified studies. A second author $(\mathrm{PJB})$ reviewed $10 \%$ of randomly selected titles and abstracts to increase the rigour of the study selection process. Uncertainty or disagreement on study selection for full-text review were resolved by a third reviewer (GE). Full-text review of selected studies was conducted by PS and GE, and disagreements resolved by a health economist $(\mathrm{CON})$.

\section{Data extraction}

Data were extracted into a standard case report form that included: author and year of publication, study design, location and setting, description of patient population, study sample size, sample demographics, data collection period, intervention description (including timing and mode of delivery), type and perspective of economic analysis (societal, healthcare system or organisation, patient, clinician), time horizon, estimated cost/resource use to implement the PDA, study-specified primary and secondary outcomes and estimated savings.

\section{Assessment of study quality}

A number of quality assessment tools were used to assess various study designs. We used the Cochrane 'risk of bias' tool to assess the quality of the included RCTs (PS and GE). ${ }^{11}$ For every one of the seven domains we judged high, low or unclear risk of bias. ${ }^{11}$ Disagreements were resolved by PJB.

We used the National Heart, Lung and Blood Institute (NHLBI) scoring checklist ${ }^{12}$ to assess the quality of the pre-post studies. The NHLBI is a 12-item questionnaire with a response format of yes (1), no (-1) or can't tell (0). A score between -12 and -4 indicates the study quality is poor, -3 to 4 is considered fair quality and $>5$ represents a good quality study. ${ }^{12}$ The Newcastle-Ottawa Quality Assessment scale $^{13}$ was used to evaluate observational studies and contains three domains judged to be poor, fair or good quality: the selection of the study groups, the comparability of the groups and the ascertainment of either the exposure or outcome of interest.

Two health economists (CON and GEC) independently used the Drummond checklist and the Consolidated Health Economic Evaluation Reporting Standards (CHEERS) checklist to assess the quality of the economic evaluations. $^{1415}$ The Drummond checklist is 10 items and indicates the quality of the economic evaluation, ${ }^{14}$ and the CHEERS' 24 items assesses the quality of how the economic evaluation was reported. ${ }^{15}$ Use of the two checklists was recommended by our health economists to ensure a comprehensive assessment of both the quality and reporting of the economic evaluations in our review. In particular, the CHEERS checklist enabled us to identify the components of the economic evaluation that were missing. For the Drummond checklist, reviewers gave a rating of 'yes' (1), 'cannot tell' $(0.5)$ or 'no' $(0) .{ }^{14}$ Higher ratings (maximum of 10 ) indicates higher quality. ${ }^{14}$ Similarly, for the CHEERS checklist, reviewers provided a score of ' 1 ' if present, ' 0 ' if absent or in some cases, 'not applicable'. ${ }^{15}$ Reviewers (CON and GEC) resolved any disagreements by discussion.

\section{Analysis}

Due to the heterogeneous nature of the reporting of economic data, we examined various indicators such as, but not limited to, the mean PDA cost per user (from any perspective), the incremental cost-effectiveness ratio or the incremental net monetary benefit. Currency data were converted to US dollars on 30 August 2019. We did not conduct a meta-analysis due to the heterogeneity of both research methods and economic analyses.

\section{Patient and public involvement}

This research was done without patient involvement. Patients were not invited to comment on the study design and were not consulted to develop patient relevant outcomes or interpret the results. Patients were not invited to contribute to the writing or editing of this document for readability or accuracy.

\section{RESULTS}

\section{Characteristics of included studies}

Our search identified 5066 studies, with 3539 remaining after the removal of duplicates. After review of their titles and abstracts, we identified 72 articles for full-text assessment; 58 did not meet the inclusion criteria. We therefore included 14 articles in addition to the 8 articles from the previous review ${ }^{2}$ for a total of 22 studies. ${ }^{16-37}$ See figure 1.

Of the 22 included studies, 16 were RCTs, 5 pre-post studies and 1 retrospective cohort study. Over 100000 participants were recruited across the studies, covering the following health conditions: breast cancer, hip and knee osteoarthritis, menorrhagia, benign prostatic hyperplasia (BPH), chest pain, cardiovascular disease, back pain, lumbar spinal stenosis, perimenopausal women, couples waiting for in vitro fertilisation, women with previous caesarean operations, residents in a geriatric health facility, decisions regarding mechanical ventilation and about the MMR (measles,mumps and rubella) vaccine acceptance. The format of the PDAs included paper $(n=4)$, web-based applications $(n=6)$ or the tool was embedded as a component of a larger intervention (ie, coaching, telephone call, DVDs, interviews) $(n=11)$. Most studies $(n=12)$ were conducted in the USA, four from the UK, two in the Netherlands and one study from each of the following countries: Australia, Canada, Finland and Japan. See table 1. 


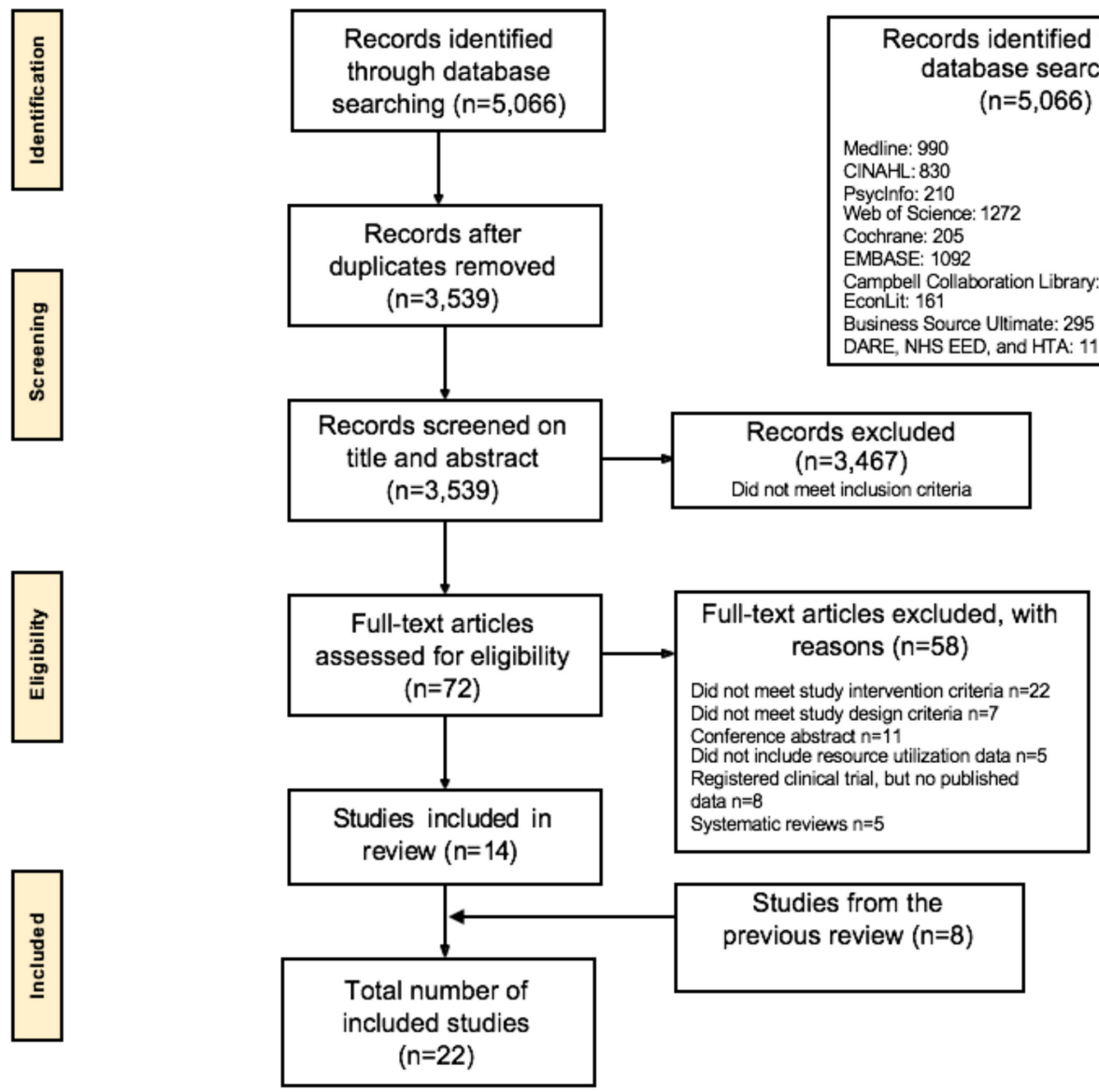

Figure 1 Flow diagram outlining the study selection process.

\section{Assessment of study quality}

For the 16 RCTs, over $70 \%$ had low risk of selection bias, over $65 \%$ had low risk of attrition bias and nearly $40 \%$ had low risk of detection or performance bias (online supplemental table $1 \mathrm{C}$ ). Pre-post studies were rated to be of fair or good quality (range $=4$ to 8 ) (online supplemental table D), and the one observational study was deemed to be of fair quality (online supplemental table E).

\section{Synthesis of evidence to determine whether PDAs generate savings \\ PDA interventions that generated cost savings}

Twelve studies reported that the PDA intervention generated cost-savings which ranged from US $\$ 10$ to US $\$ 81156$ (when adjusted to US dollars in 2020). Despite reporting that the PDA intervention generated savings, 7 of the 12 studies contained methodological issues or overstated results which impacted the interpretative validity of conclusions (see the next section for more details).

To summarise, Kennedy ${ }^{16}$ found that the interview group had lower mean costs than the control group or information-only group. Wennberg ${ }^{17}$ found that an enhanced coaching intervention for patients with preference-sensitive conditions led to a decrease in hospitalisations and significant monthly savings to the payer of healthcare services (US\$8 per member). van Peperstraten's ${ }^{18}$ empowerment strategy which included a PDA to help couples decide how many embryos should be transferred during the in vitro fertilisation process, led to mean cost savings of US $\$ 219$ per couple from the healthcare system's perspective due mainly to the lower rate of twin pregnancies in the intervention arm of the trial. Arterburn's ${ }^{19}$ decision support intervention for knee and hip replacement surgery was associated with decreased surgery rates and a reduction in arithmetic mean costs for the Group Health organisation of $17 \%$ and $19 \%$ per patient respectively. Cox's ${ }^{20}$ decision aid for mechanical ventilation were associated with a significant decrease in cost for the intervention group compared with usual care (US\$110609 vs US\$178618) which authors presume is due to fewer days in the intensive care unit, fewer hospital and ventilator days. Wilson's ${ }^{22}$ cost-benefit analysis found that the incremental net benefit (INB) of eliciting questions/ concerns of breast cancer patients pre-visit by telephone compared with doing so in person was positive (US\$65). Keyserling's ${ }^{23}$ web-based intervention to educate patients on their risk of coronary heart disease cost significantly less to implement compared with the counsellor format (US\$220 vs US\$393 less per participant) from the societal perspective when accounting for the labour (wages and market value for staff time) and non-labour (postage, printing, laptops and so on) costs. Tubeuf ${ }^{24}$ concluded that a web-based tool for first time parents whose first-born was offered the MMR vaccine had an approximate $72 \%$ 


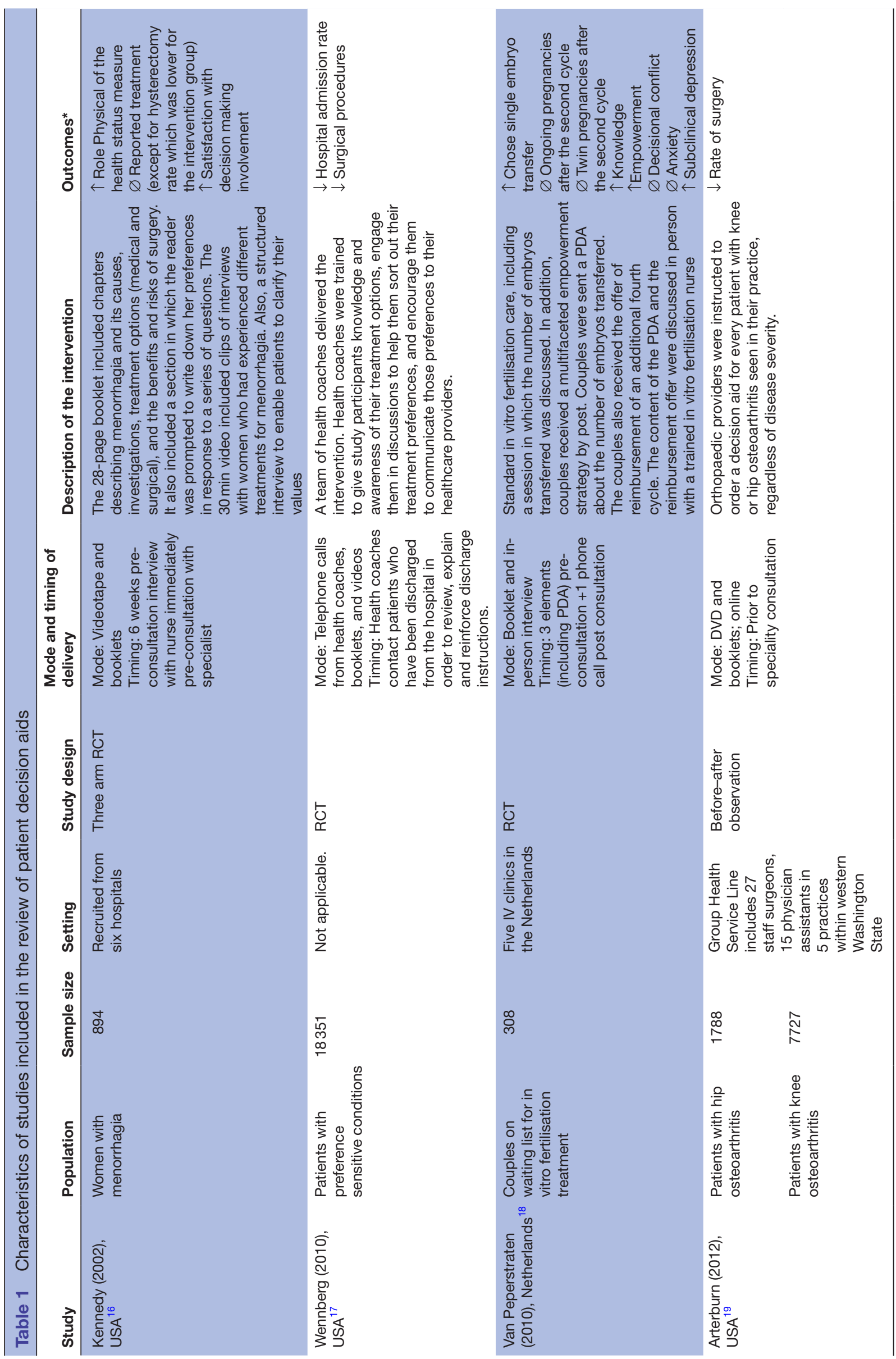




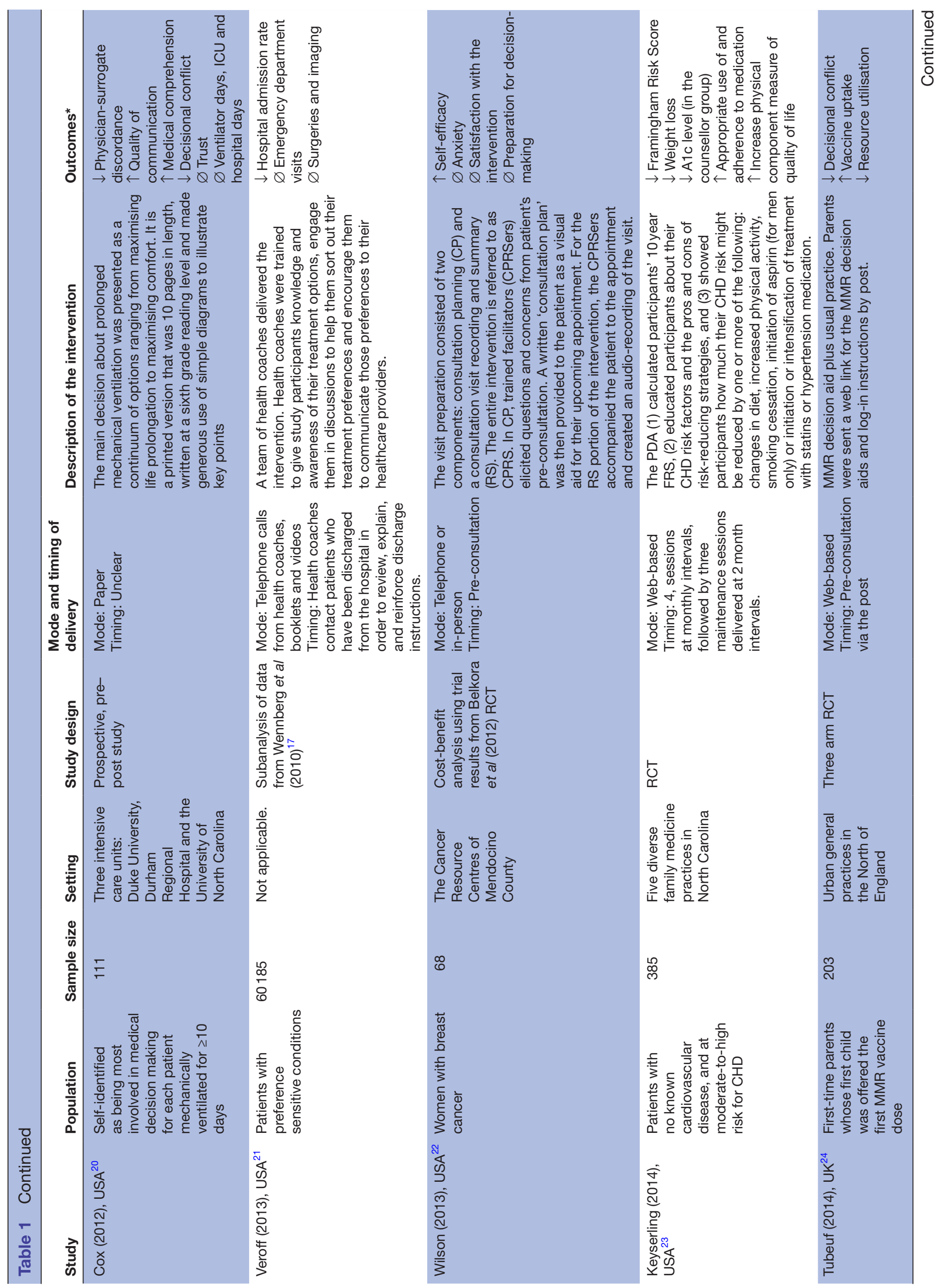




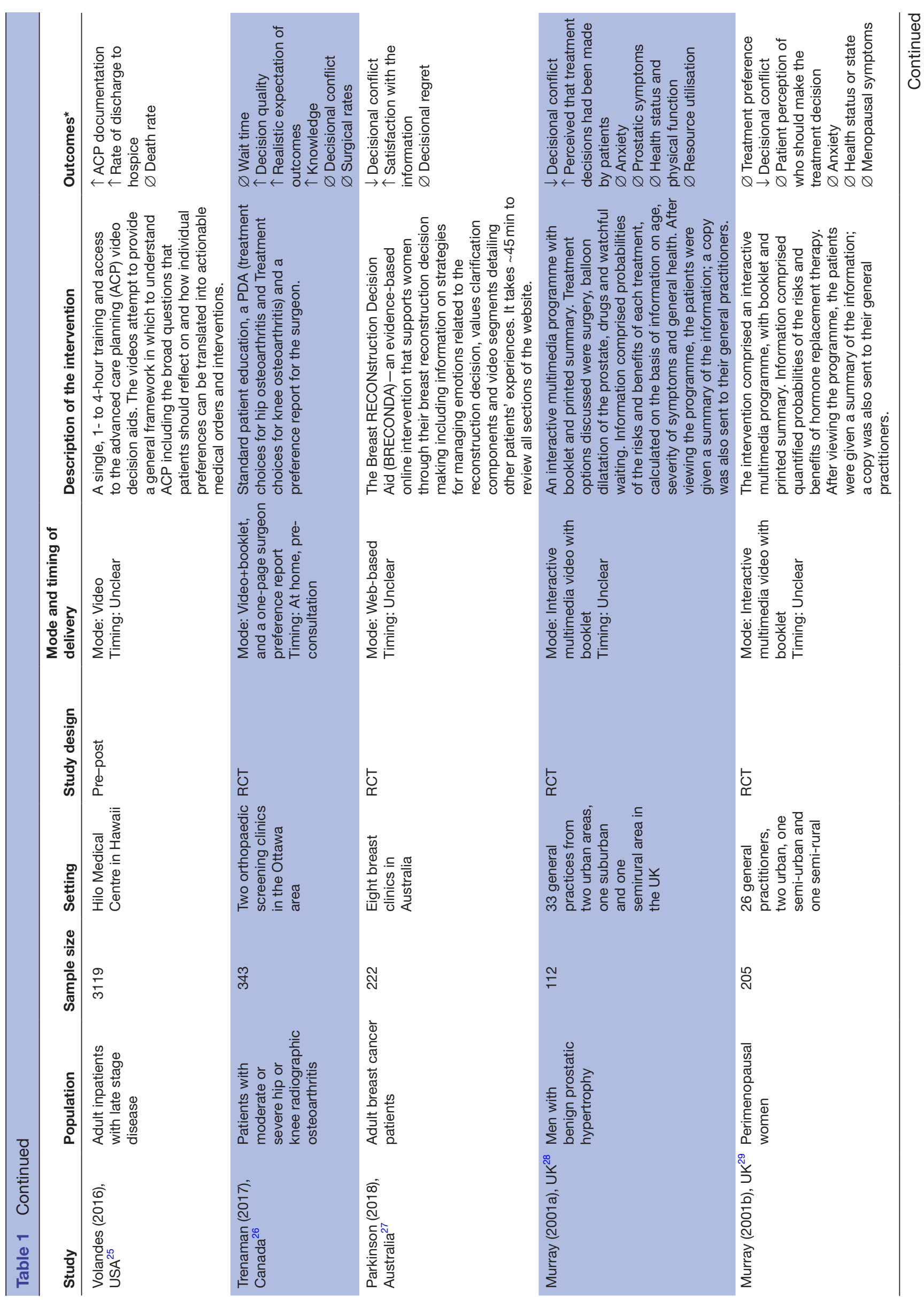




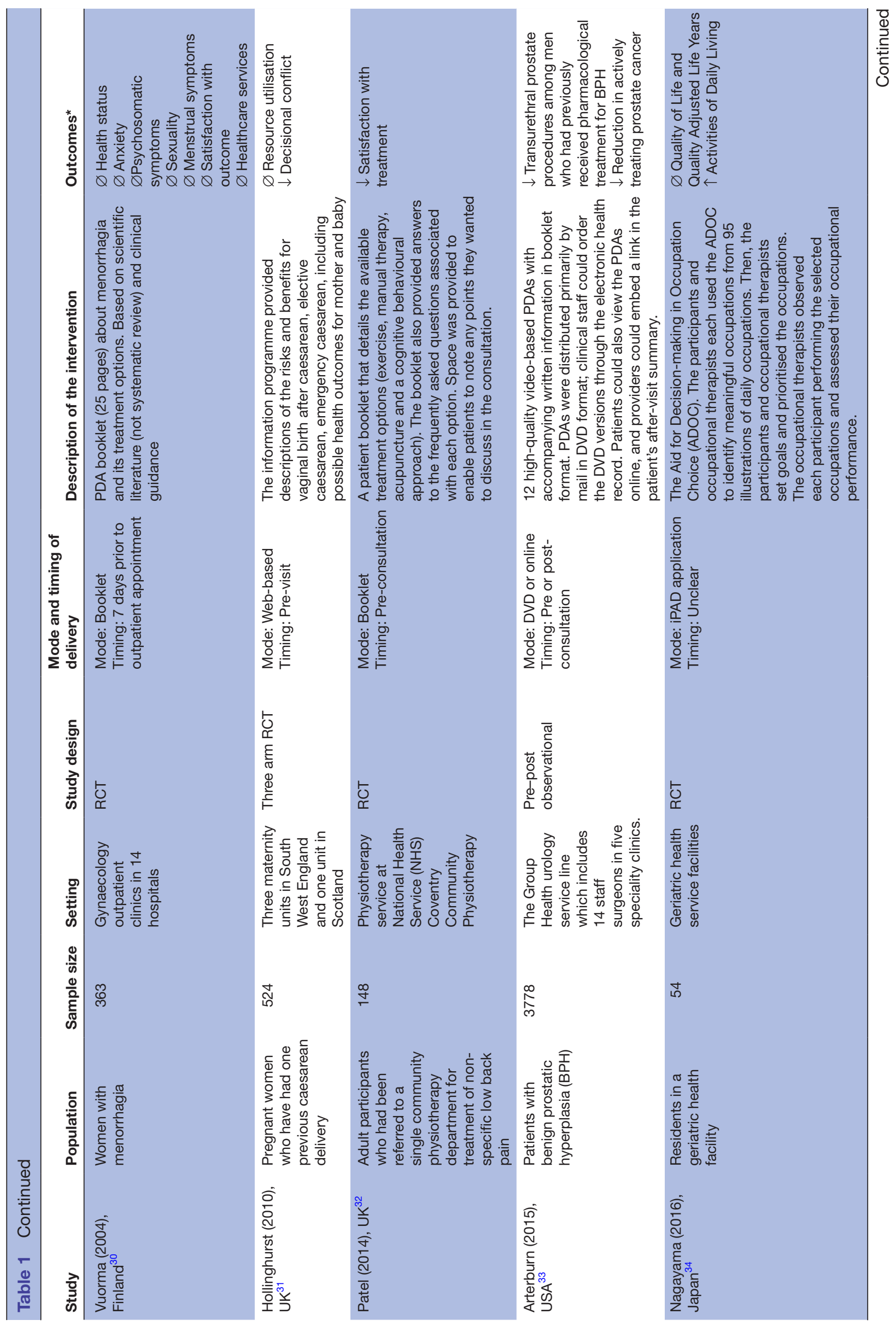




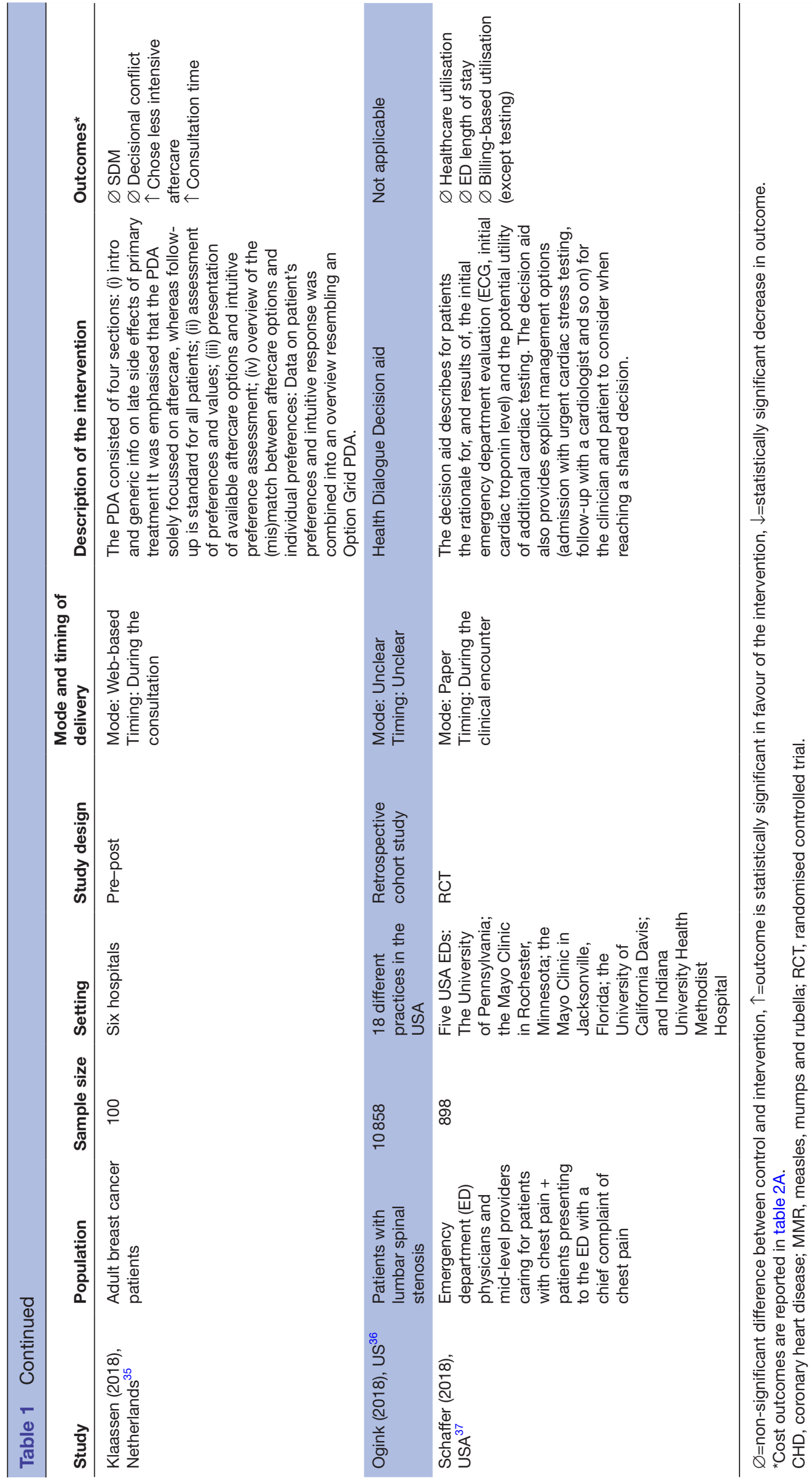


chance of being cost-effective from the National Health Service perspective. Volandes ${ }^{25}$ concluded that advanced care planning videos 'decreased healthcare costs in the last month of life for decedents' relative to the control group. Trenaman's ${ }^{26}$ cost-effectiveness analysis found that a PDA for total joint arthroplasty had a high probability of being cost-effective, ranging from $88 \%$ to $99 \%$ across willingness to pay values of US $\$ 0$ to US $\$ 100000$ per quality-adjustedlife year (QALY). Parkinson's ${ }^{27}$ online tool for breast reconstruction surgery cost less compared with usual care from the healthcare systems perspective mainly due to lower hospitalisation costs.

\section{PDA interventions that did not generate cost savings}

Ten studies reported that the PDA intervention either did not generate significant cost-savings or actually cost more than the comparator. In contrast to the previous paragraph, however, the majority of these studies (7 out of 10) expressed their conclusions conservatively based on our assessment.

To summarise, Murray's ${ }^{28} 29$ multimedia PDAs (benign prostatic hypertrophy and hormone replacement therapy) increased costs from the healthcare system perspective mainly due to the cost of the video technology. The cost of Vuorma's ${ }^{30}$ decision aid intervention to help women with menorrhagia make decisions did not generate significant savings when compared with usual care (€2760 vs $€ 3094)$, respectively from the societal perspective when accounting for surgical procedures or other medical treatments, visits, tests, the cost of producing the intervention and the personal costs to the participant. Hollinghurst's ${ }^{31}$ information programme which provided the risks and benefits for vaginal birth after caesarean cost more than usual care or decision analysis group (£2069, $£ 2033$ and £2019, respectively) from the National Health Service (NHS) perspective mainly due to the cost associated with the mode of baby delivery. Patel's ${ }^{32}$ decision support package to help patients with low back pain was not cost-effective in comparison to usual care (£264.7 vs $£ 271$, respectively) from the healthcare systems perspective when considering the cost of NHS services, tests, drugs and the cost of the intervention. ${ }^{32}$ Arterburn's ${ }^{33}$ video-based PDA for BPH and prostate cancer lowered surgery rates but was not linked to significant changes in healthcare costs from the healthcare system perspective. Nagayama $^{34}$ found that the iPAD application for Occupation Choice did not generate savings compared with standard occupational therapy (US $\$ 11643$ vs US $\$ 11$ 393, respectively) from the participants' perspective. Klaassen's ${ }^{35}$ breast cancer aftercare decision aid did not significantly reduce costs $(€ 92)$ compared with usual care $(€ 123)$ from the hospital's perspective when taking into account tests, days in the hospital, emergency room visits, physiotherapy and social work sessions though this excluded consultation time, when significantly increased. Ogink's ${ }^{36}$ retrospective cohort study found that a decision aid received by 82 patients with lumbar spinal stenosis did not change healthcare costs from the payer's perspective.
Schaffer ${ }^{37}$ evaluated the impact of the Chest Pain Choice PDA on healthcare utilisation, finding this to be lower, however the authors did not examine costs.

\section{Quality assessment of the economic analyses}

There was considerable variation in the quality of the economic analyses reporting. CHEERS scores (online supplemental table F) ranged from 50\% to $100 \%$ (mean=78\%). Key elements that were not generally reported include the incremental costs and outcomes (mean values or the main categories of estimated costs and outcomes of interest, as well as the mean differences between the comparator groups), and the characterisation of uncertainty and heterogeneity. The quality of the studies also varied widely. Drummond quality scores ranged from 0 to 10 (mean=5.4). The majority of studies did not identify the incremental analysis or relevant costs and consequences for each alternative, the allowance for uncertainty in the estimates of costs or the issues and concerns with the results. See table 2A and online supplemental table G.

Many studies contained methodological problems the most common of which were a short duration of follow-up, underpowered analyses due to small sample size or lack of a control group which affects the interpretative validity of their conclusions. To summarise: in the Wennberg ${ }^{17}$ study, and the subanalysis by Veroff, ${ }^{21}$ there was insufficient duration of follow-up. It was not possible to isolate the impact of the PDA on cost-savings as it was delivered concurrently with behavioural change and motivation counselling. A longer time horizon (2 years compared with 1 year) was reported by Trenaman ${ }^{26}$ - the only study with a perfect Drummond score-in order to assess the value for money of patient decisions aids. The observational study by Arterburn ${ }^{19}$ had no concurrent control population, no evidence on whether patients who received the decision aid via mail actually viewed them or whether patient-clinician conversations changed as a result of the decision aid, a short follow-up period (180 days) for patients undergoing elective knee and hip replacement surgery and so the association between the implementation of the decision aid and the decrease in surgical procedure rates needs to be interpreted with caution. The Keyserling ${ }^{23}$ study lacked a usual care arm, so to suggest that the web-intervention is cost-effective is misleading. Wilson ${ }^{22}$ presented the incremental net benefit as positive despite the net benefit for the two methods of delivering the intervention (telephone and in-person) being negative. Due to this presentation, the wrong impression was created that one method of delivery (telephone) was not as costly as the other (in-person), and thus warrants caution. ${ }^{22}$ In the Tubeuf ${ }^{24}$ study, costs were estimated based on intended rather than actual resource use. Conclusions from the van Peperstraten ${ }^{18}$ RCT should be treated with caution as well because the offer of reimbursement for an additional fourth cycle was only applicable if couples chose single embryo transfer in the first and second cycle and no pregnancy occurred, 


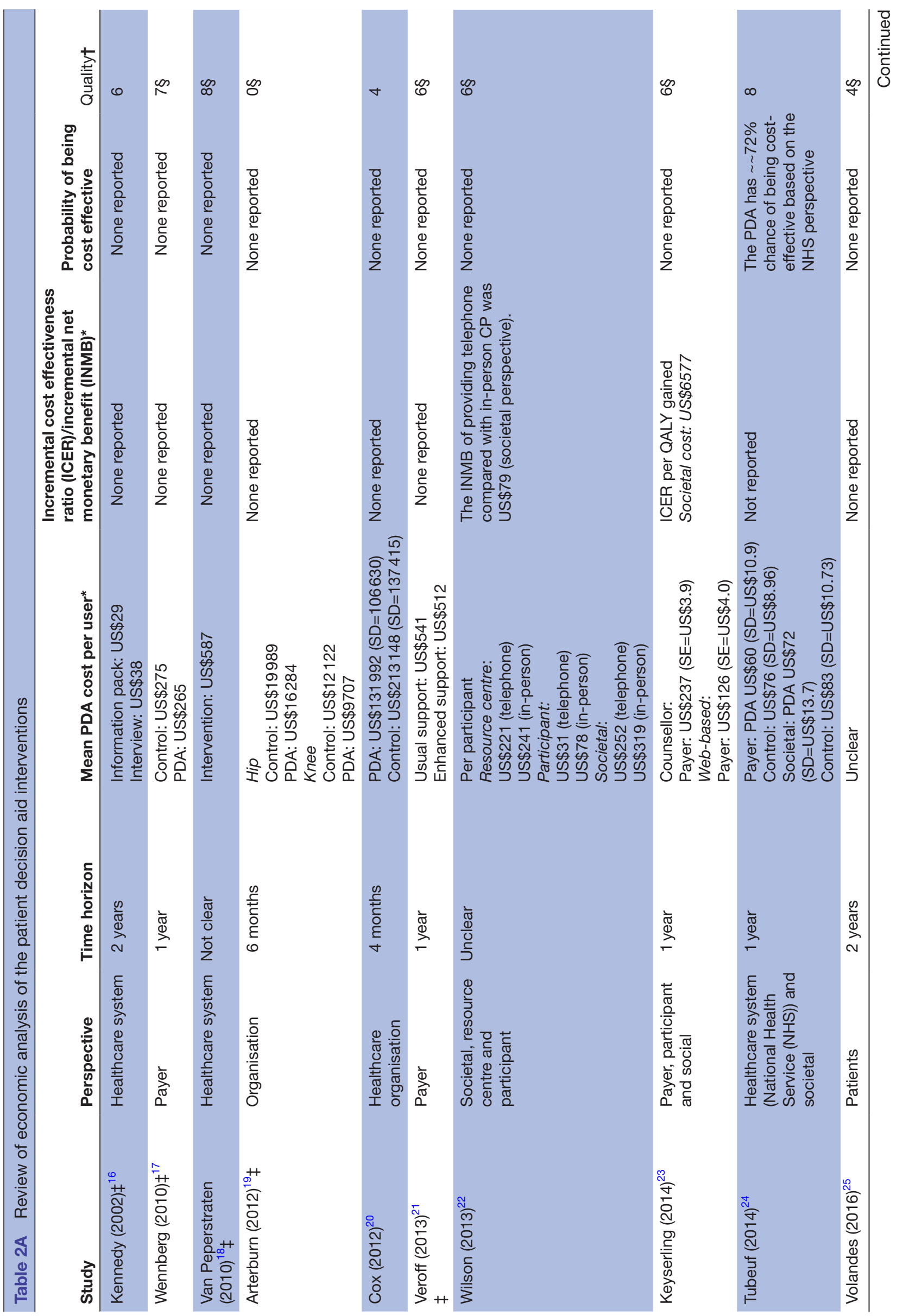




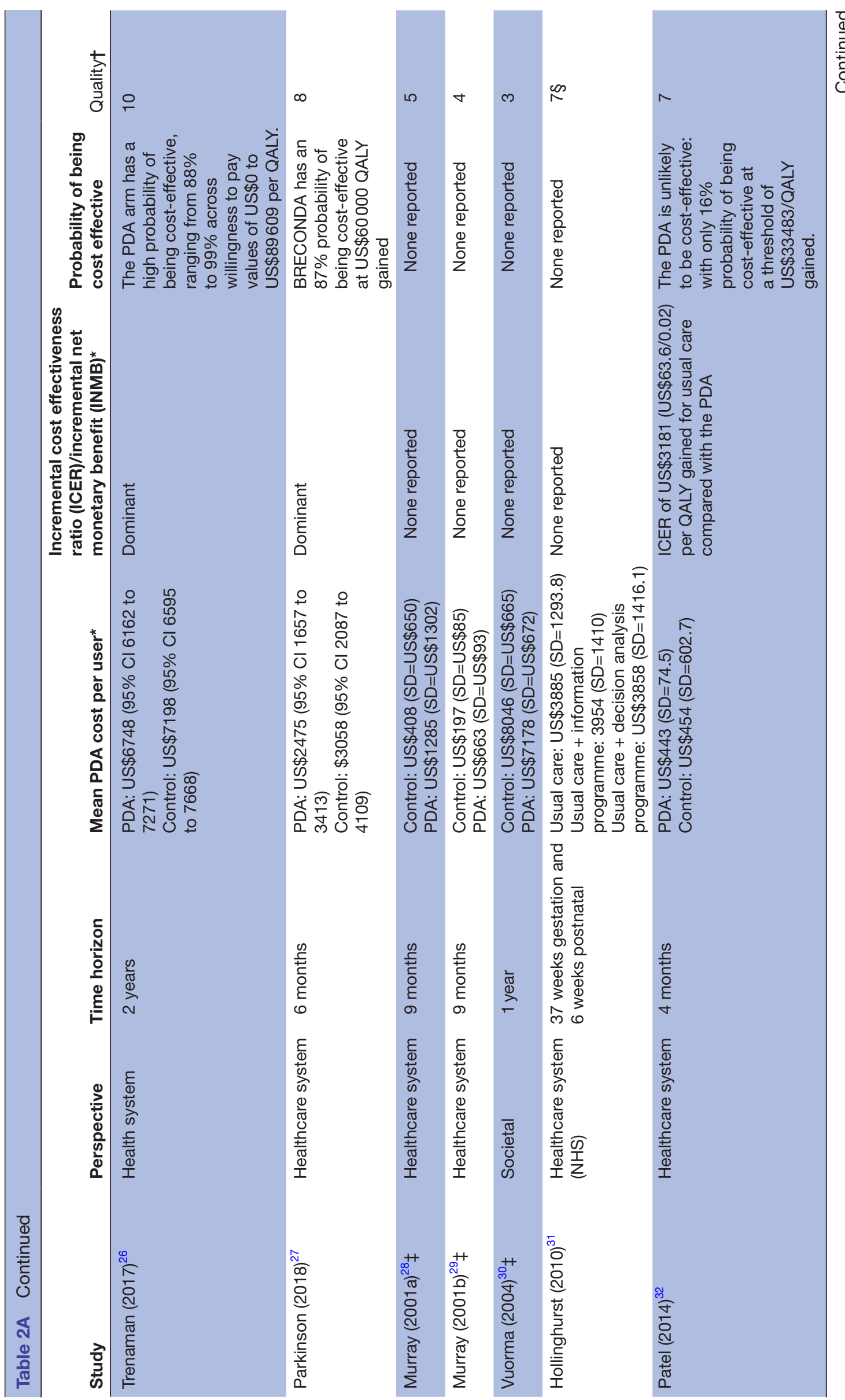

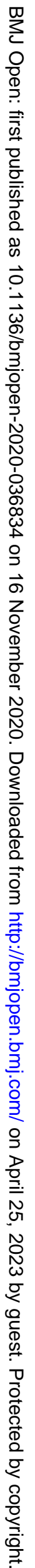




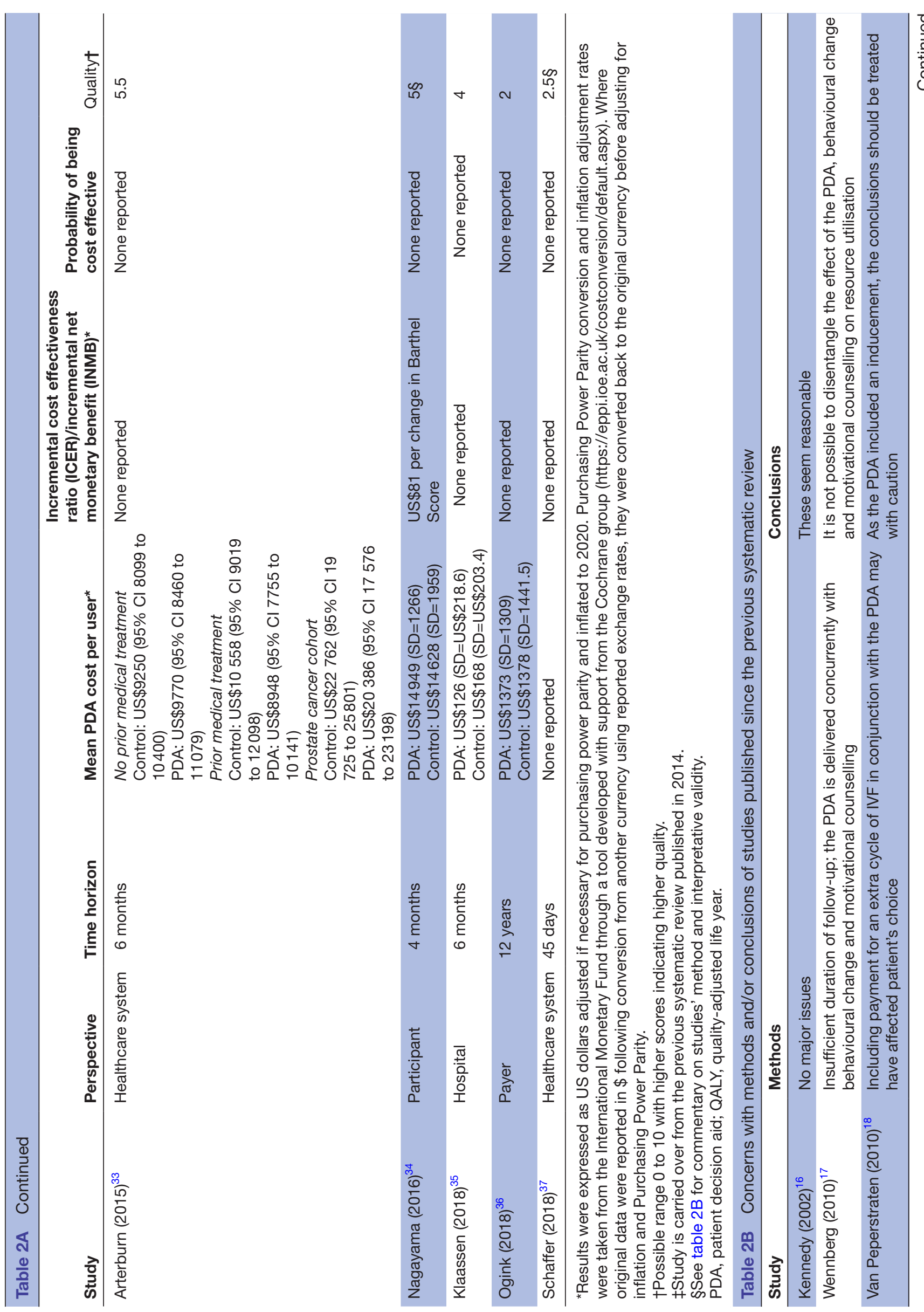

민 
which may have made the option more attractive. In addition, the costs associated with complications for the child beyond 6 weeks post-delivery (risks that may relate to delivery mode) in the Hollinghurst ${ }^{31}$ study were excluded and weighted average costs were used for delivery mode complications. See table 2B for details.

The four pilot studies, ${ }^{202533} 35$ included in this review lacked power to provide compelling evidence of costeffectiveness. The pilot studies and some other ${ }^{2033-36}$ RCTs did not include the cost of decision aids in their analyses. These reasons provide a basis on which to question any findings related to cost-savings given that the accuracy of estimated incremental costs is unclear. For instance, in the study by Parkinson, ${ }^{27}$ PDA costs assumed that everyone eligible to access the service would use the tool over the course of 3 years. This seems overly optimistic and could result in an underestimate of incremental costs. ${ }^{27}$ The methodological concerns and interpretative validity of the results are reported in table $2 \mathrm{~B}$.

\section{DISCUSSION}

\section{Statement of principal findings}

Based on our assessment of these 22 studies, and their methodological weaknesses, we conclude that there is contradictory evidence as to whether PDA use will lead to cost savings and that it is not possible to arrive at a firm conclusion. A lack of consensus exists with respect to which resources should be included when costing a PDA and apportioning these appropriately (the development, updating and maintenance of, for example, a web resource). There is also a high degree of variability with respect to: the mode of delivery for the PDA (web-based only, web-based plus interaction with a healthcare professional), the timing of delivery (before, during or after the consultation), whether the tools were actually used by the patient, the complexity of the tools' content, the subsequent compliance with the initial decision (follow-up times did not allow assessment of whether the decision merely postponed treatment) which could have positive or negative downstream cost implications, and the lack of data availability regarding the long-term impact on health-related quality of life and survival. Essentially, the heterogeneity of the methods employed due to the lack of consensus on an evaluative economic framework to assess PDAs complicates the task of determining whether or not these tools generate savings or the context in which they could generate savings.

\section{Strengths and weaknesses of the study}

Our systematic review had a number of strengths: we followed the PRISMA reporting guidelines and best practice guidelines for conducting a systematic review; two information scientists (PJB and HBB) adapted our search strategy for each database; we included a broader inclusion criteria in comparison to the EEPRU report by Ara et $a l^{10}$ which resulted in more studies and additional insight into the challenges of undertaking economic evaluations 
of PDAs; two health economists (CON and GEC) independently used two checklists to assess the quality of the economic analyses and how they were reported and evaluated the interpretative validity of the studies.

In terms of limitations, despite our best efforts we may not have found every relevant study. We believe this limitation was mitigated, however, by our search strategy which included all papers from the previous review ${ }^{2}$ and our broad inclusion criteria which included studies with non-randomised designs and the review of the studies' reference lists. The intervention descriptions were often limited, so it was difficult to ascertain whether they were PDAs. The heterogeneity of the methods and results did not enable a meta-analysis.

\section{Results in context}

Our findings mirror the conclusions of the previous systematic review (2014) which determined that the evidence as to whether or not PDAs generate savings is inconclusive due to the heterogeneous nature of the methods, the lack of quality economic analyses and the issues related to the study design and interpretation of results. ${ }^{2}$ The variation in the economic models used to assess cost in these studies, however, suggests that an agreed on economic evaluative framework needs to be developed.

The lack of consensus on an economic evaluative framework that is best applied to this research question makes it difficult to determine the context in which PDAs could generate savings. Researchers may therefore need to re-think how to approach these evaluations and how we attribute monetary value to PDAs. Butt described the limitations of the current economic frameworks because they focus on 'health gain' when in fact the PDAs may have a much broader impact. ${ }^{8}$ Butt states that 'by excluding other benefits within the broad umbrella of process of care, the utility of decision aids is likely to be undervalued'. ${ }^{8}$ Other variables such as the duration of the patient-clinician relationship, the health literacy of the patient, the timing and mode of delivery may impact monetary value and clinical encounter duration. ${ }^{8}$ For this reason, the consultation time trade-off (CTTO) has been proposed as a new evaluative framework to assess the cost-effectiveness of PDAs. ${ }^{8}$ The CTTO would represent 'the number of minutes the patient would be willing to trade for use of the tool', so the number of consultation minutes saved due to the PDA would be converted to a monetary value using the clinician's wage rate. ${ }^{8}$

Furthermore, the EEPRU (2015) suggested that the 'the scope of an economic evaluation of PDAs needs to be extended beyond the health-related QALY' to include the wider societal benefits of health services. ${ }^{10}$ The report suggests that finding ways to express the benefits such as increased knowledge, reduced decisional conflict and improved patient-clinician communication in terms of QALYs or another metric in which value is captured is important in any cost estimation. ${ }^{10}$ Ultimately, the development of a framework which could potentially include non-health outcomes in the valuation of PDAs could lead to more well-designed economic evaluations and more definitive conclusions about the impact of these tools on cost and outcomes. The adoption of a welfarist costbenefit approach in which stated or revealed preferences are expressed in monetary terms and related to costs may offer a way forward

\section{Implications}

The lack of definitive evidence that PDAs lead to costsavings can be attributed to a number of factors. Existing studies lack sufficient methodological rigour and are of limited duration given the potential consequences of these tools may extend over time. ${ }^{38}$ Current evaluative frameworks may be too narrow, and therefore limit the potential to consider the full scope of impact on savings.

Ultimately, economic evaluations provide a comparative analysis of alternative uses of resources in terms of cost and outcomes. The technique is well established, and clear guidance on the conduct and reporting of evaluations exists including identification of appropriate comparators, study perspective (which will determine costs included) and choice of appropriate outcome. However, the sine qua non for an evaluation of a PDA is consensus around the definition of a PDA, including its delivery modality. While there remains ambiguity in the evaluation literature around what constitutes a PDA, assessments of their cost-effectiveness and comparisons of these will continue to be problematic. Ideally, an evaluation should first set out how the intervention described meets accepted criteria for its consideration as a PDA. Only then are the questions of which PDAs, and under which circumstances, provide good value for money likely to be answered in a convincing manner.

It is also important to voice the argument that supporting patients and clinicians to arrive at informed, well-considered decisions has value in and of itself. This value has been recognised by many organisations including NICE (National Institute for Health and Care Excellence) in the UK who are committed to placing patient involvement at the fore of the treatment decisionmaking process. ${ }^{39}$ Similarly, the Affordable Care Act advocated that shared decision making be supported by the development of certified PDAs-an indication of the value of engaging patients in decisions. ${ }^{40}$ What monetary value do we place on high quality decisions-on doing the right thing? It is a question that lies at the heart of current health systems.

\section{CONCLUSION}

It is unclear based on the quality of the economic evidence available to date, whether PDAs generate cost savings in healthcare settings, regardless of the perspective being analysed. Nevertheless, the evidence that these tools improve patient outcomes and the overall quality of decision-making should encourage their implementation in practice by organisations who wish to practice 
patient-centred care. Going forward it is important to conduct robust and well-designed economic evaluations which have sufficient follow-up to enable costs and outcomes (both health and non-health) to be assessed over longer time horizons.

\section{Twitter Paul J Barr @BarrPaulJ and Glyn Elwyn @glynelwyn}

Contributors PS, GEC and PJB developed the search strategy, and reviewed studies to determine if they met the inclusion criteria. PJB and HBB adapted the search for the selected databases and conducted the search. PS extracted data. CON and GE applied two checklists to rate the reporting and quality of the economic data included in each study. All authors contributed to the manuscript and approved the final version.

Funding The authors have not declared a specific grant for this research from any funding agency in the public, commercial or not-for-profit sectors.

Competing interests GE has edited and published books that provide royalties on sales by the publishers: the books include Shared Decision Making (Oxford University Press) and Groups (Radcliffe Press). He has in the past provided consultancy for organisations including (1) Emmi Solutions LLC who developed patient decision support tools; (2) National Quality Forum on the certification of decision support tools; (3) Washington State Health Department on the certification of decision support tools; and (4) SCiMentu LLC, Amsterdam (workshops for shared decision making). He is the Founder and Director of \&think LLC which owns the registered trademark for Option Grids TM patient decision aids. Founder and director of SHARPNetwork LLC, a provider of training for shared decision making. He provides advice in the domain of shared decision making and patient decision aids to (1) Access Community Health Network, Chicago Federally Qualified Medical Centers; (2) EBSCO Health Option Grids TM patient decision aids; (3) Bind Insurance; (4) PatientWisdom Inc; and (5) abridge Al Inc. GE academic interests are focussed on shared decision making and coproduction. He owns copyright in measures of shared decision making and care integration namely collaboRATE, integRATE, consideRATE, coopeRATE, toleRATE, Observer OPTION-5 and Observer OPTION-12.

\section{Patient consent for publication Not required}

Provenance and peer review Not commissioned; externally peer reviewed.

Data availability statement All data relevant to the study are included in the article or uploaded as supplementary information. No additional data are available. The manuscript's guarantor (GE) affirms that this manuscript is an honest, accurate and transparent account of the study being reported; that no important aspects of the study have been omitted; and that any discrepancies from the study as planned (and, if relevant, registered) have been explained.

Supplemental material This content has been supplied by the author(s). It has not been vetted by BMJ Publishing Group Limited (BMJ) and may not have been peer-reviewed. Any opinions or recommendations discussed are solely those of the author(s) and are not endorsed by BMJ. BMJ disclaims all liability and responsibility arising from any reliance placed on the content. Where the content includes any translated material, BMJ does not warrant the accuracy and reliability of the translations (including but not limited to local regulations, clinical guidelines, terminology, drug names and drug dosages), and is not responsible for any error and/or omissions arising from translation and adaptation or otherwise.

Open access This is an open access article distributed in accordance with the Creative Commons Attribution Non Commercial (CC BY-NC 4.0) license, which permits others to distribute, remix, adapt, build upon this work non-commercially, and license their derivative works on different terms, provided the original work is properly cited, appropriate credit is given, any changes made indicated, and the use is non-commercial. See: http://creativecommons.org/licenses/by-nc/4.0/.

\section{ORCID iDs}

Peter Scalia http://orcid.org/0000-0002-5050-435X

Glyn Elwyn http://orcid.org/0000-0002-0917-6286

\section{REFERENCES}

1 Stacey D, Légaré F, Lewis K, et al. Decision AIDS for people facing health treatment or screening decisions. Cochrane Database Syst Rev 2017;4:CD001431.
2 Walsh T, Barr PJ, Thompson R, et al. Undetermined impact of patient decision support interventions on healthcare costs and savings: systematic review. BMJ 2014;348:g188.

3 Härter M, Moumiid N, Cornuz J, et al. Shared decision making in 2017: international accomplishments in policy, research and implementation. Z Evid Fortbild Qual Gesundhwes 2017;123124:1-5.

4 Coulter A. National strategies for implementing shared decision making. Bertelsmann Stifftung 2018:1-68.

5 Scalia P, Durand M-A, Berkowitz JL, et al. The impact and utility of encounter patient decision AIDS: systematic review, meta-analysis and narrative synthesis. Patient Educ Couns 2019:102:817-41.

6 Légaré F, Adekpedjou R, Stacey D, et al. Interventions for increasing the use of shared decision making by healthcare professionals. Cochrane Database Syst Rev 2018;7:CD006732.

7 Trenaman L, Bryan S, Bansback N. The cost-effectiveness of patient decision AIDS: a systematic review. Health Care 2014;2:251-7.

8 Butt T. Measuring the benefits of decision AIDS for economic evaluation. Pharmacoecon Open 2019;3:143-50.

9 Methley AM, Campbell S, Chew-Graham C, et al. PICO, PICOS and spider: a comparison study of specificity and sensitivity in three search tools for qualitative systematic reviews. BMC Health Serv Res 2014;14:579.

10 Ara R, Brazier JE, Sculpher M, et al. A framework for conducting economic evaluations when using patient decision AIDS in health care decision making. Policy Research Unit in Economic Evaluation of Health and Care Interventions 2015;049:1-49.

11 Cumpston M, Li T, Page MJ, et al. Updated guidance for trusted systematic reviews: a new edition of the Cochrane Handbook for systematic reviews of interventions. Cochrane Database Syst Rev 2019;10:ED000142.

12 National Heart, Lung, and Blood Institute. Study quality assessment tools, 2018. Available: https://www.nhlbi.nih.gov/health-topics/studyquality-assessment-tools

13 Ottawa Hospital Research Institute. The Newcastle-Ottawa scale (NOS) for assessing the quality of nonrandomized studies in metaanalyses, 2019. Available: http://www.ohri.ca/programs/clinical_ epidemiology/oxford.asp

14 Drummond MF, Sculpher MJ, Claxton K, et al. Methods for the economic evaluation of health care programmes. Oxford university press, 2015.

15 Husereau D, Drummond M, Petrou S, et al. Consolidated health economic evaluation reporting standards (cheers) statement. Value in Health 2013;16:e1-5.

16 Kennedy ADM, Sculpher MJ, Coulter A, et al. Effects of decision AIDS for menorrhagia on treatment choices, health outcomes, and costs. JAMA 2002;288:2701-8.

17 Wennberg DE, Marr A, Lang L, et al. A randomized trial of a telephone care-management strategy. N Engl J Med 2010;363:1245-55

18 van Peperstraten A, Nelen W, Grol R, et al. The effect of a multifaceted empowerment strategy on decision making about the number of embryos transferred in in vitro fertilisation: randomised controlled trial. BMJ 2010;341:c2501.

19 Arterburn D, Wellman R, Westbrook E, et al. Introducing decision AIDS at group health was linked to sharply lower hip and knee surgery rates and costs. Health Aff 2012;31:2094-104

20 Cox CE, Lewis CL, Hanson LC, et al. Development and pilot testing of a decision aid for surrogates of patients with prolonged mechanical ventilation*. Crit Care Med 2012;40:2327-34.

21 Veroff D, Marr A, Wennberg DE. Enhanced support for shared decision making reduced costs of care for patients with preferencesensitive conditions. Health Aff 2013;32:285-93.

22 Wilson L, Loucks A, Stupar L, et al. Cost-Benefit analysis of decision support methods for patients with breast cancer in a rural community. Commun Oncol 2013;10:47-57.

23 Keyserling TC, Sheridan SL, Draeger LB, et al. A comparison of live counseling with a web-based lifestyle and medication intervention to reduce coronary heart disease risk. JAMA Intern Med 2014:174:1144-57.

24 Tubeuf S, Edlin R, Shourie S, et al. Cost effectiveness of a webbased decision aid for parents deciding about MMR vaccination: a three-arm cluster randomised controlled trial in primary care. $\mathrm{Br} \mathrm{J}$ Gen Pract 2014;64:e493-9.

25 Volandes AE, Paasche-Orlow MK, Davis AD, et al. Use of Video Decision Aids to Promote Advance Care Planning in Hilo, Hawai'i. $J$ Gen Intern Med 2016;31:1035-40.

26 Trenaman L, Stacey D, Bryan S, et al. Decision AIDS for patients considering total joint replacement: a cost-effectiveness analysis alongside a randomised controlled trial. Osteoarthritis Cartilage 2017;25:1615-22. 
27 Parkinson B, Sherman KA, Brown P, et al. Cost-effectiveness of the $B R E C O N D A$ decision aid for women with breast cancer: Results from a randomized controlled trial. Psychooncology 2018;27:1589-96.

28 Murray E, Davis H, Tai SS, et al. Randomised controlled trial of an interactive multimedia decision aid on benign prostatic hypertrophy in primary. BMJ 2001;323:493-6.

29 Murray E, Davis H, Tai SS, et al. Randomised controlled trial of an interactive multimedia decision aid on hormone replacement therapy in primary. BMJ 2001;323:490-3.

30 Vuorma S, Teperi J, Aalto A-M, et al. A randomized trial among women with heavy menstruation - impact of a decision aid on treatment outcomes and costs. Health Expect 2004;7:327-37.

31 Hollinghurst S, Emmett C, Peters TJ, et al. Economic evaluation of the diamond randomized trial: cost and outcomes of 2 decision AIDS for mode of delivery among women with a previous cesarean section. Med Decis Making 2010;30:453-63.

32 Patel S, Ngunjiri A, Hee SW, et al. Primum non nocere: shared informed decision making in low back pain--a pilot cluster randomised trial. BMC Musculoskelet Disord 2014;15:282.

33 Arterburn D, Wellman R, Westbrook EO, et al. Decision AIDS for benign prostatic hyperplasia and prostate cancer. Am J Manag Care 2015;21:e130-40.

34 Nagayama H, Tomori K, Ohno K, et al. Effectiveness and costeffectiveness of occupation-based occupational therapy using the aid for decision making in occupation choice (ADOC) for older residents: pilot cluster randomized controlled trial. PLoS One 2016;11:e0150374.

35 Klaassen LA, Dirksen CD, Boersma LJ, et al. A novel patient decision aid for aftercare in breast cancer patients: a promising tool to reduce costs by individualizing aftercare. The Breast 2018;41:144-50.

36 Ogink PT, Teunis T, van Wulfften Palthe $\mathrm{O}$, et al. Variation in costs among surgeons for lumbar spinal stenosis. The Spine Journal 2018;18:1584-91.

37 Schaffer JT, Hess EP, Hollander JE, et al. Impact of a shared decision making intervention on health care utilization: a secondary analysis of the chest pain choice multicenter randomized trial. Acad Emerg Med 2018;25:293-300.

38 Elwyn G, Frosch DL, Kobrin S. Implementing shared decisionmaking: consider all the consequences. Implementation Sci 2015; $11: 114$.

39 National Institute for health and care excellence (NICE). NICE guidelines, 2019. Available: https://www.nice.org.uk/about/what-wedo/our-programmes/nice-guidance/nice-guidelines/shared-decisionmaking

40 Durand M-A, Barr PJ, Walsh T, et al. Incentivizing shared decision making in the USA--where are we now? Healthc 2015;3:97-101. 